\title{
Performance Analysis of CSTR for Different Minimization Routines of NNMPC
}

\author{
Sanjay Baweja \\ PhD Scholor \\ Dept. of Chemical Engineering \\ Career Point Univ., Kota-324005, \\ Rajasthan, india
}

\author{
Rajeev Gupta \\ Prof. \& Pro Vice Chancellor \\ Dept. of Electronics Engineering \\ Raj. Tech. Univ. Kota-324010, \\ Rajasthan, India
}

\begin{abstract}
Continues Stirred Tank Reactor (CSTR) is widely used in chemical industries and to get high productivity and quality from CSTR the control of various parameter is an important issue. Neural Network based Model Predictive Controller (NNMPC) refers to a class of control algorithms that compute a sequence of manipulated variable adjustments in order to optimize the future behaviour of a plant. In the present study NNMPC is implemented in Neural Network Toolbox of Matlab software that calculates the control input to optimize CSTR performance over a specified future time horizon using minimization routines based on five different line searches. These five conjugate gradient based line searches are namely, Golden section; Bent's; Hybrid bisection cubic; Charalambous and Backtracking line searches. Performance analysis of CSTR output response and error convergence plot indicates that the brent's line search based minimization routine gives best result as compared to other line searches and the NNMPC utilizing Brent's line search based minimization routine controls the output concentration effectively.
\end{abstract}

\section{Keywords}

Continuous stirred tank reactor, Matlab, Model predictive control, neural network, System Identification.

\section{INTRODUCTION}

The traditional process control systems uses linear dynamic models whereas for the highly nonlinear systems, control techniques which are based on nonlinear models may provide significant improvement. Model Predictive Control (MPC) concept is being extensively studied and widely acceptable in industrial applications. The main reasons for such popularity of the predictive control strategies are the intuitiveness and the explicit constraint handling.

Adaptive tracking control is considered for a class of general nonlinear systems using multilayer neural networks(MNNs)[1]. A non linear model of the plant is approximated by an external model, and the resultant controller ensures internal properties and stability of the control system[2]. Non-linear control of CSTR for reversible reaction is carried out using Neural Network as design tool[3]. Fuzzy optimal control methodology is applied to the design of the feedback loops of an Exothermic Continuous Stirred Tank Reactor system[4].Developing a model incorporating the nonlinear dynamics of the system warrants lot of computation, an efficient control of the product concentration can be achieved only through accurate model.[5].Neural Network Approximate Generalized Predictive Control (NNAPC) that uses a combination of Artificial Neural Network (ANN) with Approximate Generalized Predictive Control technique (APC).[6]. Fuzzy based structure strategy gives the more flexibility and precise behavior in control action in comparison to the least square based approach.[7]. Predictive control algorithm is applied to control the concentration in a continuous stirred tank reactor (CSTR), whose parameters are optimally determined by solving quadratic performance index using the optimization algorithm[8].

Another approach to neuro-fuzzy control is a combination of the neural predictive controller and the neuro-fuzzy controller (Adaptive Network-based Fuzzy Inference System - ANFIS). These controllers work in parallel. The output of ANFIS adjusts the output of the neural predictive controller to enhance the control performance. Such design of an intelligent control system is applied to control of the continuous stirred tank reactor and laboratory mixing process[9]. Adaptive controller is an efficient controller for temperature control of CSTR than PID controller[10].The simulation result shows that both NNPC and SVMPC gives better control performance than PID for set-point change as well as for load change of $\pm 10 \%$ in methanol feed flow rate and molar ratio of methanol to isoamylene in reactor effluent feed[11]

The control is performed in primary and secondary controlloops where the primary controlled output of the reactor is a concentration of the main reaction product and the secondary output is the reactant temperature. A common control input is the coolant flow rate. The controller in the primary controlloop is a P-controller with an adjustable gain. A controller in the secondary control-loop consist of the static nonlinear and the dynamic adaptive linear part. The proposed method is verified by control simulations[12]. For controlling the product composition in the CSTR, the neuro controller NARMA-L2 is implemented by manipulating the input feed composition. The simulation results show the superiority of the NARMA-L2 in accurately tracking the composition setpoint changes in the CSTR and control the system better as compared to that of the conventional PID[13].

Literature review of earlier work clearly indicates that the application of NN for CSTR output control is attempted several times before, but none of the author included the effect of design parameters of $\mathrm{NN}$ on the performance of CSTR. Thus an attempt had been made in the presented paper to analyze these effects.

In this paper, introduction is given in section I. The remainder of the paper is organized as follows: In Section II, the Neural Network based Model Predictive control(NNMPC) is described. Section III discuss various minimization routines, section IV explains CSTR non-linear Plant Model and section $\mathrm{V}$ provides simulation model for validation and testing of the designed controller. The results and discussions are given in Section VI, and section VII presents concluding remarks.

2. NN MODEL PREDICTIVE CONTROL The neural network model predictive controller uses a neural network model of a nonlinear plant to predict future plant 
performance. The controller then calculates the control input that will optimize plant performance over a specified future time horizon. The first step in model predictive control is to determine the neural network plant model (system identification). Next, the plant model is used by the controller to predict future performance(optimization process).

\subsection{System Identification}

The first stage of model predictive control is to train a neural network to represent the forward dynamics of the plant. The prediction error between the plant output and the neural network output is used as the neural network training signal. The neural network plant model uses previous inputs and previous plant outputs to predict future values of the plant output. The structure of the neural network plant model consists of input layer, hidden layers and an output layer. This network can be trained offline in batch mode, using data collected from the operation of the plant. Training is done in batch mode using fast algorithms that uses one of the three standard numerical optimization techniques i.e. Conjugate gradient; Quasi-Newton and Levenberg-Marquardt (trainlm).

The Levenberg-Marquardt algorithm [14-16]is used in the present paper as it appears to be the fastest method for training moderate-sized feedforward neural networks (up to several hundred weights). It also has a very efficient MATLAB implementation, because the solution of the matrix equation is a built-in function, so its attributes become even more pronounced in a MATLAB setting.

\subsection{Predictive Control}

The model predictive control method is based on the receding horizon technique [17]. The neural network model predicts the plant response over a specified time horizon. The predictions are used by a numerical optimization program to determine the control signal that minimizes the following performance criterion over the specified horizon

$=\sum_{j=N_{1}}^{N_{2}}\left(y_{r}(t+j)-y_{m}(t+j)\right)^{2}+\rho \sum_{j=1}^{N_{u}}\left(u^{\prime}(t+j-1)-\right.$ $\left.u^{\prime}(t+j-2)\right)$

where $N_{1}, N_{2}$, and $N_{u}$ define the horizons over which the tracking error and the control increments are evaluated. The $u^{\prime}$ variable is the tentative control signal, $y_{r}$ is the desired response, and $y_{m}$ is the network model response. The $\rho$ value determines the contribution that the sum of the squares of the control increments has on the performance index.

Fig.1 illustrates the block diagram for model predictive control process. The controller consists of the neural network plant model and the optimization block. The optimization block determines the values of $u^{\prime}$ that minimize $J$, and then the optimal $u$ is input to the plant. The controller block is implemented in Simulink, as described in the section V.

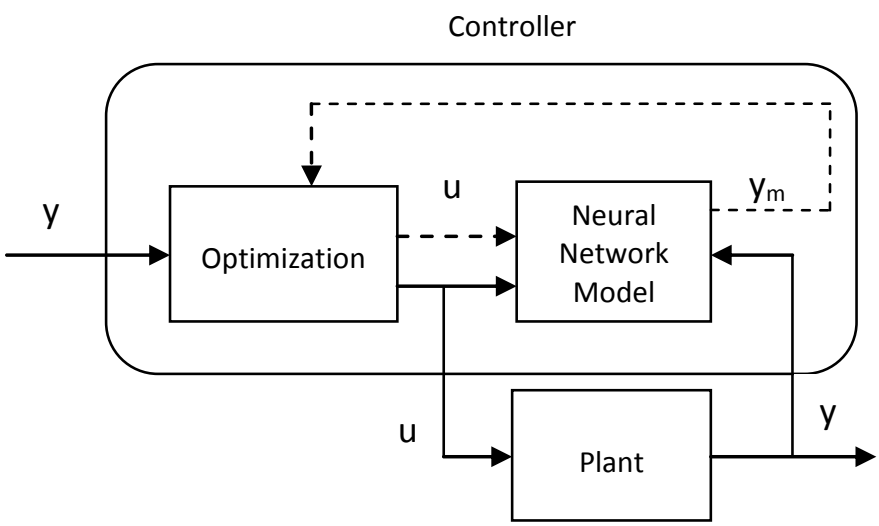

Fig.1 Block diagram of model predictive control process

\section{MINIMIZATION ROUTINES}

Optimization process involves minimization of error using minimization routines based on conjugate gradient based line search algorithms. In most of the conjugate gradient algorithms, the step size is adjusted at each iteration. A search is made along the conjugate gradient direction to determine the step size, which minimizes the performance function along that line. Five different search functions are discussed here that are used to analyze the performance of plant(CSTR) output :

\subsection{Golden Section Search (srchgol)}

The golden section search srchgol is a linear search that does not require the calculation of the slope. This routine begins by locating an interval in which the minimum of the performance occurs. This is accomplished by evaluating the performance at a sequence of points, starting at a distance of delta and doubling in distance each step, along the search direction. When the performance increases between two successive iterations, a minimum has been bracketed. The next step is to reduce the size of the interval containing the minimum. Two new points are located within the initial interval. The values of the performance at these two points determines a section of the interval that can be discarded, and a new interior point is placed within the new interval. This procedure is continued until the interval of uncertainty is reduced to a width of tolerance of search, which is equal to step size/Parameter that relates the tolerance to the initial step size.

\subsection{Brent's Search (srchbre)}

Brent's search is a linear search, which is a hybrid combination of the golden section search and a quadratic interpolation. Function comparison methods, like the golden section search, have a first-order rate of convergence, while polynomial interpolation methods have an asymptotic rate that is faster than superlinear. On the other hand, the rate of convergence for the golden section search starts when the algorithm is initialized, whereas the asymptotic behavior for the polynomial interpolation methods may take many iterations to become apparent. Brent's search attempts to combine the best features of both approaches.

For Brent's search the same interval of uncertainty is kept as it is with golden section search, but some additional points are computed. A quadratic function is then fitted to these points and the minimum of the quadratic function is computed. If this minimum is within the appropriate interval of uncertainty, it is used in the next stage of the search and a new quadratic approximation is performed. If the minimum falls outside the 
known interval of uncertainty, then a step of the golden section search is performed.

\subsection{Hybrid Bisection-Cubic Search (srchhyb)}

Like Brent's search, srchhyb is a hybrid algorithm. It is a combination of bisection and cubic interpolation. For the bisection algorithm, one point is located in the interval of uncertainty and the performance and its derivative are computed. Based on this information, half of the interval of uncertainty is discarded. In the hybrid algorithm, a cubic interpolation of the function is obtained by using the value of the performance and its derivative at the two end points. If the minimum of the cubic interpolation falls within the known interval of uncertainty, then it is used to reduce the interval of uncertainty. Otherwise, a step of the bisection algorithm is used.

\subsection{Charalambous' Search (srchcha)}

The method of Charalambous srchcha was designed to be used in combination with a conjugate gradient algorithm for neural network training, it is a hybrid search. It uses a cubic interpolation, together with a type of sectioning.

\subsection{Backtracking (srchbac)}

The backtracking search routine srchbac is best suited to use with the quasi-Newton optimization algorithms. It begins with a step multiplier of 1 and then backtracks until an acceptable reduction in the performance is obtained. On the first step it uses the value of performance at the current point and at a step multiplier of 1 . Also it uses the value of the derivative of performance at the current point, to obtain a quadratic approximation to the performance function along the search direction. The minimum of the quadratic approximation becomes a tentative optimum point (under certain conditions) and the performance at this point is tested. If the performance is not sufficiently reduced, a cubic interpolation is obtained and the minimum of the cubic interpolation becomes the new tentative optimum point. This process is continued until a sufficient reduction in the performance is obtained.

\section{PLANT MODEL}

A standard catalytic Continuous Stirred Tank Reactor (CSTR) model is used in the present study. The diagram of the process is shown in the Fig.2

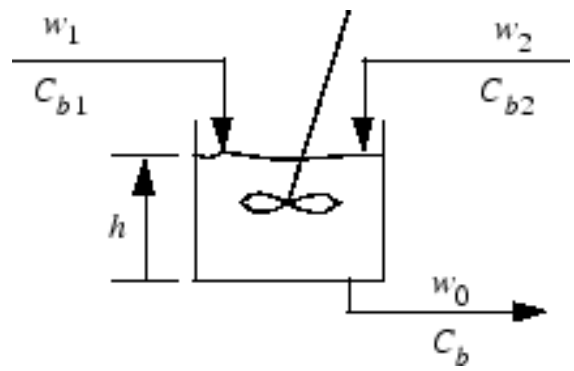

Fig. 2 Plant Model[18]

The dynamic model of the system is

$$
\begin{aligned}
& \frac{d(h)}{d t}=w_{1}(t)+w_{2}(t)-0.2 \sqrt{h(t)} \\
& \frac{d c_{b}(t)}{d t}=\left(c_{b 1}-c_{b}(t)\right) \frac{w_{1}(t)}{h(t)}+\left(c_{b 2}-c_{b}(t)\right) \frac{w_{2}(t)}{h(t)}- \\
& \frac{k_{1} c_{b}(t)}{\left(1+k_{2} c_{b}(t)\right)^{2}}
\end{aligned}
$$

where $h(t)$ is the liquid level, $C_{b}(t)$ is the product concentration at the output of the process, $w_{1}(t)$ is the flow rate of the concentrated feed $C_{b 1}$, and $w_{2}(t)$ is the flow rate of the diluted feed $C_{b 2}$. The input concentrations are set to $C_{b 1}=24.9$ and $C_{b 2}=0.1$. The constants associated with the rate of consumption are $k_{1}=1$ and $k_{2}=1$.

The objective of the controller is to maintain the product concentration by adjusting the flow $w_{1}(t)$. To simplify the example, set $w_{2}(t)=0.1$. The level of the $\operatorname{tank} h(t)$ is not controlled for this experiment.

\section{SIMULATION MODEL}

A diagram of the simulation process is shown in Fig.3, it includes a catalytic Continuous Stirred Tank Reactor (CSTR) whose output concentration is controlled via NNPC, input to the controller is set to a random reference signal.

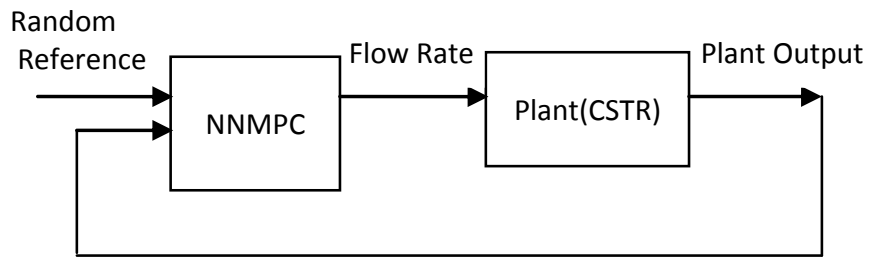

Fig.3 Block diagram of simulation model

Plant Identification is done to develop the neural network plant model to predict future plant outputs. The optimization algorithm uses these predictions to determine the control inputs that optimize future performance. The plant model neural network has seven hidden layer, the number of delayed inputs- 2 nos. and delayed outputs- 2 nos., and the training function is trainlm to train the neural network plant model. Then training data is generated which is further used to train the network(plant model) according to the training algorithm. Simulation is performed after loading the trained neural network plant model into the NN Predictive Controller block.

The NN Predictive Controller is designed by setting controller horizons $N_{2}$ and $N_{u}\left(N_{1}\right.$ is fixed at 1.), weighting parameter $\rho$, the parameter $\alpha$ to control the optimization. It determines how much reduction in performance is required for a successful optimization step. Five different minimization routines are used by the optimization algorithm, and 2 iterations of the optimization algorithm are performed at each sample time. As the simulation runs, the plant output for five different minimization routines and the random reference signal are displayed, as in the Fig.4. Also the error convergence plot is shown in Fig. 5 that compares the reference signal with 5 outputs having different minimization routines to optimize the neural network predictive controller. 


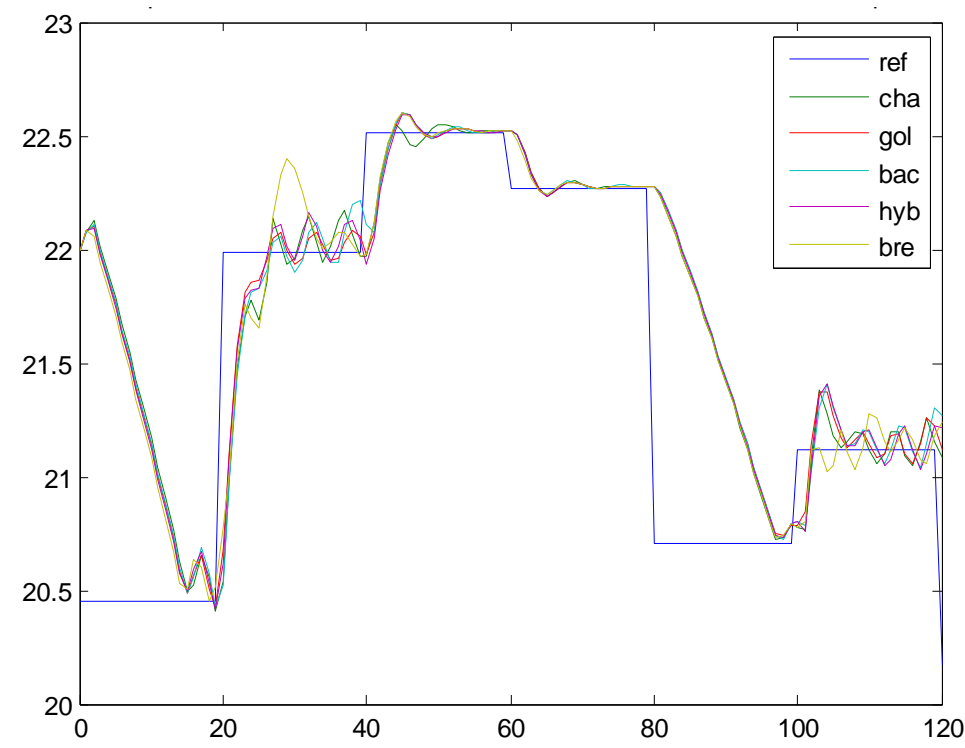

Fig.4 Output response of CSTR for different line search algorithms v/s ref. signal

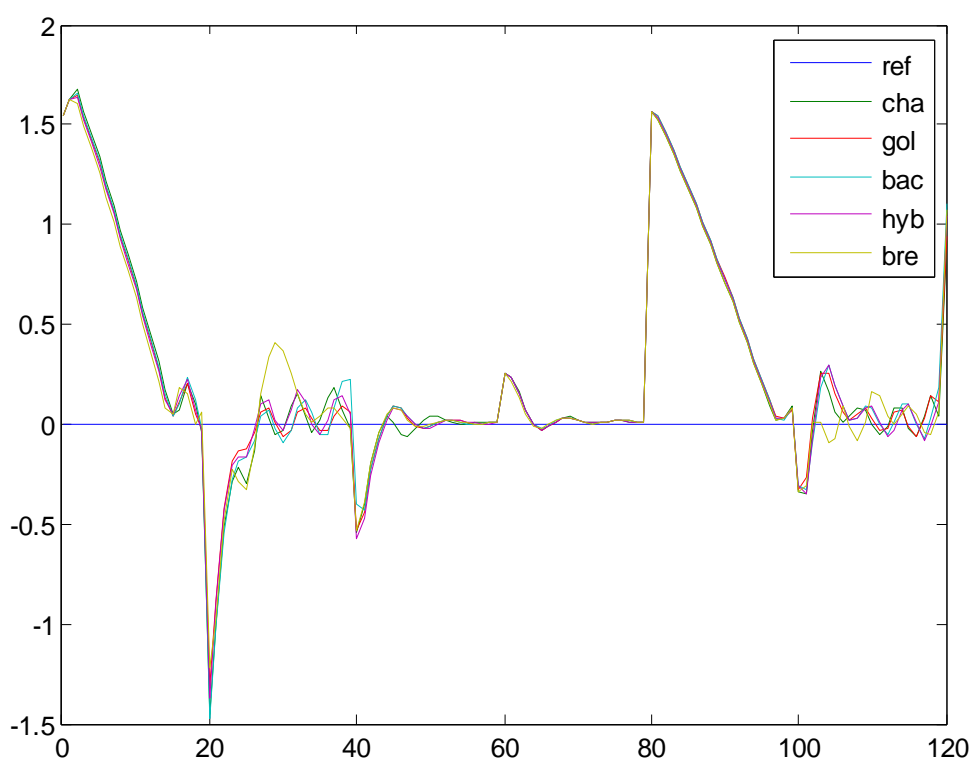

Fig.5 Error convergence plot for five different line search algorithms/minimization routines

Table 1 Comparison of statistical characteristics of five minimization routines.

\begin{tabular}{|c|c|c|c|c|c|}
\hline & \multicolumn{5}{|c|}{ Type of Minimization Routines } \\
\cline { 2 - 6 } Statistics & Brent (bre) & $\begin{array}{c}\text { Golden Section } \\
\text { (gol) }\end{array}$ & $\begin{array}{c}\text { Hybrid } \\
\text { Bisection } \\
\text { Cubic (hyb) }\end{array}$ & $\begin{array}{c}\text { Charalambous } \\
\text { (cha) }\end{array}$ & $\begin{array}{c}\text { Backtracking } \\
\text { (bac) }\end{array}$ \\
\hline Min & -1.2154 & -1.3131 & -1.3693 & -1.4534 & -1.4693 \\
\hline Max & 1.6312 & 1.6497 & 1.6380 & 1.6765 & 1.6556 \\
\hline Mean & 0.2407 & 0.2458 & 0.2440 & 0.2378 & 0.2468 \\
\hline Median & 0.0461 & 0.0387 & 0.0457 & 0.0377 & 0.0422 \\
\hline Mode & -1.2154 & -1.3131 & -1.3693 & -1.4534 & -1.4693 \\
\hline Std & 0.5263 & 0.5313 & 0.5332 & 0.5424 & 0.5423 \\
\hline Range & 2.8466 & 2.9628 & 3.0073 & 3.1299 & 3.1249 \\
\hline
\end{tabular}

\section{RESULTS AND DISCUSSIONS}

Fig.4 shows the output concentration response of CSTR corresponding to different line searches used for optimization of NNMPC. Five different line search algorithms are used for minimisation of MSE during the conjugate-gradient based training of the multilayered feed forward neural network 
based predictive controller. Fig.5 indicates the error convergence in the five conditions, using data statistics tool in Matlab a comparison is obtained for the five conditions. Table 1 gives the comparison of statistical parameters for various line search routines and shows that the Brent's line search routine has minimum magnitude of range, mode and standard deviation, hence it is best suited for the particular application.

\section{CONCLUSION}

Application of neural network predictive control for output concentration control of continuous stirred tank reactor had been successfully attempted. Designing of NNMPC using multilayer feedforward neural network involves conjugate gradient based training. Training of neural network is done by minimizing the mean square error(MSE) cost function using different line search algorithms. Out of the five line searches attempted, the brent's line search based minimization routine train the neural network more accurately as compared to other line searches. It has minimum standard deviation and appears closer to the random reference signal. Thus it can be concluded from the present study that Brent's line search based minimization routine outperforms in the present scenario and significantly affect the output of CSTR. In future the effect of other NNMPC design parameters can be analyzed for CSTR application.

\section{REFERENCES}

[1] Ge, S.S., Hang, C.C., and Zhang T.1993. Nonlinear adaptive control using neural networks and its application to CSTR systems, Journal of Process Control, 9, 313-323.

[2] Bakosova, M., Bobal, V. and Dosta,1 P. 2004. An approach to adaptive control of a CSTR, chem. Pap. 58(3) 184-190.

[3] Afolab, T. J., Aloko, D., and Araromi, D. O., .2007. Neural Network Control of CSTR for Reversible Reaction Using Reverence Model Approach, Leonardo Journal of Sciences, 10, 25-40.

[4] Boudeghdegh, K., Khellaf, A., Leulmi, S. and Soukkou1, A.2008. Neural Network Control of CSTR for Reversible Reaction usingreverence Model Approach, , Brazilian Journal of Chemical Engineering,. 25(4), $799-812$.

[5] Suja Mani Malar, R. and. Thyagarajan, T. 2009. Artificial Neural Networks Based Modeling and Control of Continuous Stirred Tank Reactor, American J. of Engineering and Applied Sciences 2 (1): 229-235.

[6] Kalhoodashti, H.E.2011. Concentration Control of CSTR using NNAPC, International Journal of Computer Applications , 26(6), 34-38.
[7] Movahed, M.A, Mahmoudzadeh, S. Rahmat, M.F, and Yazdani, A.M .2011. Temperature Control of A Continuous Stirred Tank Reactor By Means Of Two Different Intelligent Strategies, International Journal On Smart Sensing And Intelligent Systems ,4(2), 244-267.

[8] Shrivastava, P. 2012. Modeling and Control of CSTR using Model based Neural Network Predictive Control, International Journal of Computer Science and Information Security, 10( 7), 38-43.

[9] Blahova, L. Dvoran, J. and Kmetova J. 2012. Neurofuzzy control design of processes in chemical technologies, Archives of Control sciences ,22(LVIII), No. 2, 233-250.

[10] Murali Bhaskaran, V. and Prabhu, K. 2014. Adaptive Controller Design for Continuous Stirred Tank Reactor, Research Journal of Applied Sciences, Engineering and Technology, 8(10), 1217-1224.

[11] Sharma, N. and Singh, K. 2014. Neural network and support vector machine predictive control of tert-amyl methyl ether reactive distillation column, Systems Science \& Control Engineering: An Open Access Journal, 2, 512-526.

[12] .Bobál, V. Dostál, P. and Vojtesek, J. 2015. Application of Cascade Nonlinear Control for a CSTR, Wseas Transactions On Systems, 14, 252-260.

[13] Abdullah, N. Mohamad, A.B, Mohamed, A. Mustafa, M.M. Osman, M.H. and Yee, T.C. 2016. Control of Continuous Stirred Tank Reactor using Neural Networks, Indian Journal of Science and Technology, 9(21), 1-7.

[14] Marquardt, D. 1963. An Algorithm for Least-Squares Estimation of Nonlinear Parameters, SIAM Journal on Applied Mathematics, 11(2), 431-441.

[15] Hagan, M.T. and Menhaj, M. 1999. "Training feedforward networks with the Marquardt algorithm," IEEE Transactions on Neural Networks, 5(6), 989-993.

[16] Hagan, M.T., Demuth, H.B. and Beale, M.H. 1996. Neural Network Design, Boston, MA: PWS Publishing.

[17] Soloway, D. and Haley, P.J. 1996. "Neural Generalized Predictive Control," Proceedings of the 1996 IEEE International Symposium on Intelligent Control, 277281.

[18] Neural Network Toolbox @ 1984-2007 The MathWorks, Inc. 